\title{
Effectiveness of the BNT162b2 mRNA COVID-19 vaccine in pregnancy
}

\author{
Noa Dagan 1,2,3,4,14, Noam Barda1,2,3,4,14, Tal Biron-Shental ${ }^{5,6}$, Maya Makov-Assif', Calanit Key ${ }^{7}$, \\ Isaac S. Kohane ${ }^{3,4}$, Miguel A. Hernán ${ }^{8,9}$, Marc Lipsitch ${ }^{10}{ }^{10}$, Sonia Hernandez-Diaz ${ }^{8}$, Ben Y. Reis ${ }^{4,11,12}$ \\ and Ran D. Balicer (10), 1,13凶
}

\begin{abstract}
To evaluate the effectiveness of the BNT162b2 messenger RNA vaccine in pregnant women, we conducted an observational cohort study of pregnant women aged 16 years or older, with no history of SARS-CoV-2, who were vaccinated between 20 December 2020 and 3 June 2021. A total of 10,861 vaccinated pregnant women were matched to 10,861 unvaccinated pregnant controls using demographic and clinical characteristics. Study outcomes included documented infection with SARS-CoV-2, symptomatic COVID-19, COVID-19-related hospitalization, severe illness and death. Estimated vaccine effectiveness from 7 through to $56 \mathrm{~d}$ after the second dose was $96 \%$ (95\% confidence interval $89-100 \%$ ) for any documented infection, $97 \%$ (91-100\%) for infections with documented symptoms and $89 \%$ (43-100\%) for COVID-19-related hospitalization. Only one event of severe illness was observed in the unvaccinated group and no deaths were observed in either group. In summary, the BNT162b2 mRNA vaccine was estimated to have high vaccine effectiveness in pregnant women, which is similar to the effectiveness estimated in the general population.
\end{abstract}

Hundreds of millions of individuals worldwide have been infected by the SARS-CoV-2 virus and millions have died from COVID-19 and related complications. Vaccines are currently the leading approach for combating the pandemic's advance. Phase 3 clinical trials conducted to evaluate the safety and efficacy of mRNA COVID-19 vaccines did not include pregnant women, even though they are at risk for severe COVID- $19^{1}$ and potentially for adverse pregnancy outcomes ${ }^{2}$. Ongoing trials ${ }^{3}$ are limited to late pregnancy vaccination and surrogate efficacy outcomes (that is, immunogenicity). Given the current lack of evidence regarding the safety and efficacy of the vaccines for this population ${ }^{4,5}$, vaccination guidelines ${ }^{6-8}$ for pregnant women have been inconsistent, ranging from contraindicated to permitted to recommended in pregnancy.

As the number of vaccinated individuals increases worldwide, there is an opportunity to evaluate the real-world effectiveness and safety of the mRNA COVID-19 vaccines using observational data. Initial reports regarding vaccine safety indicate no obvious safety signals among pregnant women'. However, information regarding vaccine effectiveness among pregnant women is still limited ${ }^{10}$.

The immune system is known to undergo alterations during pregnancy. For example, there is evidence that levels of $\mathrm{CD}^{+}$and $\mathrm{CD}^{+}$ lymphocytes decrease during pregnancy, as do the levels of some inflammatory cytokines ${ }^{11}$. Because mRNA-based vaccines are a new technology that has not been widely tested in pregnant women, it is plausible that the immune response triggered by these vaccines in pregnant women may be altered compared to the general population, increasing the need to evaluate vaccine effectiveness specifically for this subpopulation. In a previous report, confidence in vaccine effectiveness among pregnant women was mentioned as one of the strongest predictors of COVID-19 vaccine acceptance in this group ${ }^{12}$.

The objective of this study was to estimate real-world vaccine effectiveness in a large observational cohort of pregnant women, aged 16 years or older, with no previous SARS-CoV-2 infection and recruited between 20 December 2020 and 3 June 2021. Vaccinated women were exactly matched to unvaccinated controls on a set of demographic and clinical characteristics and followed for a median of $77 \mathrm{~d}$. Vaccine effectiveness was estimated in several follow-up periods for documented infection with SARS-CoV-2, symptomatic COVID-19, COVID-19-related hospitalization, severe COVID-19 and COVID-19-related death.

\section{Results}

Of 38,836 women members of Clalit Health Services (CHS) vaccinated during pregnancy, 28,227 met the eligibility criteria (Methods); 10,861 of these vaccinated women were successfully matched to unvaccinated pregnant controls (Extended Data Fig. 1). The full population was similar to the eligible population (Supplementary Table 1). Matched individuals were also similar to the eligible population, albeit with a lower prevalence of some risk factors for severe COVID-19 (Supplementary Table 1).

The baseline characteristics of the matched individuals were very similar in the vaccinated and unvaccinated groups (Supplementary Table 2). The median age was 30 years, with $26 \%$, $48 \%$ and $26 \%$ of pregnancies in the first, second and third trimesters, respectively.

${ }^{1}$ Clalit Research Institute, Innovation Division, Clalit Health Services, Tel Aviv, Israel. 2Software and Information Systems Engineering, Ben Gurion University, Be'er Sheva, Israel. ${ }^{3}$ Department of Biomedical Informatics, Harvard Medical School, Boston, MA, USA. ${ }^{4}$ The Ivan and Francesca Berkowitz Family Living Laboratory Collaboration at Harvard Medical School and Clalit Research Institute, Boston, MA, USA. ${ }^{5}$ Department of Obstetrics and Gynecology, Meir Medical Center, Kfar Saba, Israel. ${ }^{6}$ Sackler School of Medicine, Tel Aviv University, Tel Aviv, Israel. ${ }^{7}$ Clalit Community Division, Clalit Health Services, Tel Aviv, Israel. ${ }^{8}$ Departments of Epidemiology and Biostatistics, Harvard T.H. Chan School of Public Health, Boston, MA, USA. ${ }^{9}$ CAUSALab, Harvard T.H. Chan School of Public Health, Boston, MA, USA. ${ }^{10}$ Center for Communicable Disease Dynamics, Department of Epidemiology and Department of Immunology and Infectious Diseases, Harvard T.H. Chan School of Public Health, Boston, MA, USA. "Predictive Medicine Group, Computational Health Informatics Program, Boston Children's Hospital, Boston, MA, USA. ${ }^{12}$ Harvard Medical School, Boston, MA, USA. ${ }^{13 S c h o o l ~ o f ~ P u b l i c ~ H e a l t h, ~ F a c u l t y ~ o f ~}$ Health Sciences, Ben Gurion University of the Negev, Be'er Sheva, Israel. ${ }^{14}$ These authors contributed equally: Noa Dagan, Noam Barda.

凶e-mail: Rbalicer@clalit.org.il 


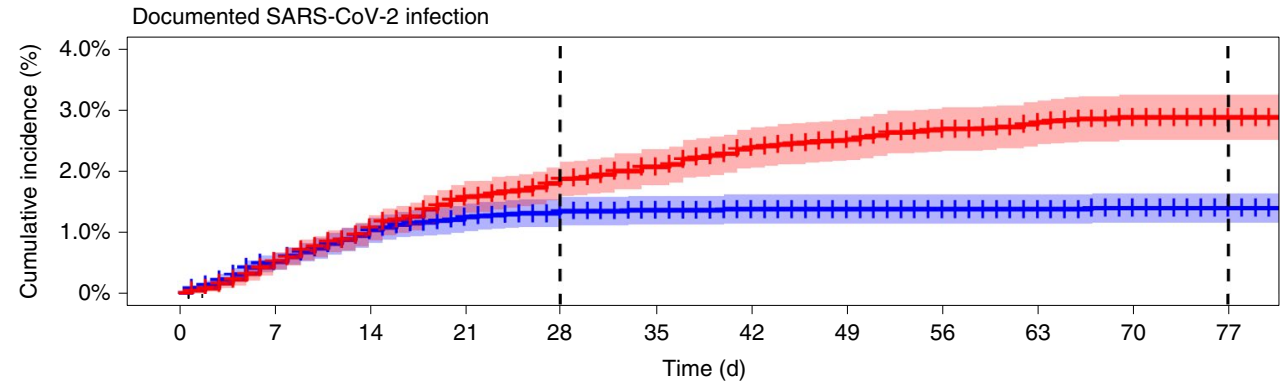

\begin{tabular}{|c|c|c|c|c|c|c|c|c|c|c|c|}
\hline $\begin{array}{l}10,861 \\
10,861\end{array}$ & $\begin{array}{l}9,877 \\
9,883\end{array}$ & $\begin{array}{l}8,933 \\
8,926\end{array}$ & $\begin{array}{l}8,225 \\
8,189\end{array}$ & $\begin{array}{l}7,577 \\
7,519\end{array}$ & $\begin{array}{l}7,008 \\
6,926\end{array}$ & $\begin{array}{l}6,561 \\
6,452\end{array}$ & $\begin{array}{l}6,113 \\
5,994\end{array}$ & $\begin{array}{l}5,761 \\
5,627\end{array}$ & $\begin{array}{l}5,342 \\
5,200\end{array}$ & $\begin{array}{l}4,961 \\
4,813\end{array}$ & $\begin{array}{l}4,508 \\
4,360 \\
\end{array}$ \\
\hline 0 & 7 & 14 & 21 & 28 & 35 & 42 & 49 & 56 & 63 & 70 & 77 \\
\hline
\end{tabular}

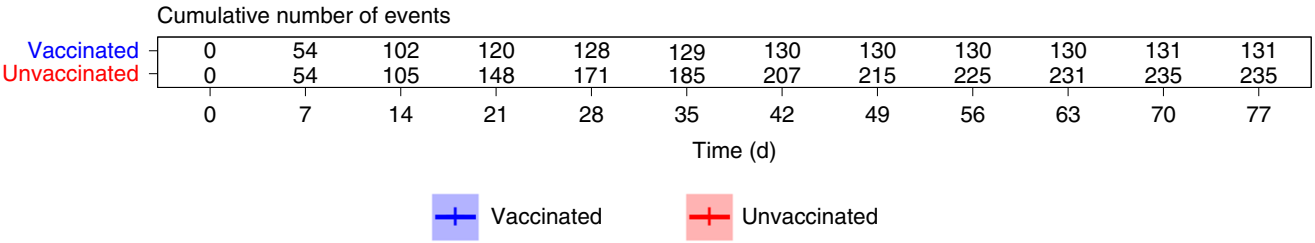

Fig. 1 | Cumulative incidence of SARS-CoV-2 documented infection in vaccinated pregnant women and matched controls. Cumulative incidence curves of the documented infection outcome in pregnant women, CHS, 20 December 2020 through to 3 June 2021 . The main line is the point estimate of the cumulative incidence and the shaded areas are the $95 \% \mathrm{Cls}$. The vertical lines at days 28 and 77 demarcate the period when an individual was considered 'fully vaccinated' in the manuscript ( 7 through to $56 \mathrm{~d}$ after receipt of the second dose). The curve represents the numbers at risk at each time point, along with the cumulative number of events. Each individual was included only once in each study group but individuals could move from the unvaccinated to the vaccinated group after receipt of the vaccine.

Table 1 | Vaccine effectiveness measures

\begin{tabular}{|c|c|c|c|c|c|c|c|c|}
\hline \multirow[t]{2}{*}{ Period } & \multicolumn{2}{|c|}{ Documented infection } & \multicolumn{2}{|c|}{ Symptomatic infection } & \multicolumn{2}{|c|}{ Hospitalization } & \multicolumn{2}{|c|}{ Severe disease } \\
\hline & $\begin{array}{l}\text { 1-RR } \\
(95 \% \mathrm{CI})\end{array}$ & $\begin{array}{l}\text { RD } \\
(95 \% \mathrm{CI})\end{array}$ & $\begin{array}{l}\text { 1-RR } \\
(95 \% \mathrm{CI})\end{array}$ & $\begin{array}{l}\text { RD } \\
(95 \% \mathrm{CI})\end{array}$ & $\begin{array}{l}\text { 1-RR } \\
(95 \% \mathrm{CI})\end{array}$ & $\begin{array}{l}\text { RD } \\
(95 \% \mathrm{Cl})\end{array}$ & $\begin{array}{l}1-\text { RR } \\
(95 \% \mathrm{CI})\end{array}$ & $\begin{array}{l}\text { RD } \\
(95 \% \mathrm{CI})\end{array}$ \\
\hline $\begin{array}{l}\text { Days } 14-20 \text { after } \\
\text { first dose }\end{array}$ & $\begin{array}{l}67 \% \\
(40-84 \%)\end{array}$ & $\begin{array}{l}309.22 \\
(145.43-485.69)\end{array}$ & $\begin{array}{l}66 \% \\
(32-86 \%)\end{array}$ & $\begin{array}{l}223.59 \\
(82.44-361.63)\end{array}$ & 3 versus $0^{a}$ & & 2 versus $0^{a}$ & \\
\hline $\begin{array}{l}\text { Days } 21-27 \text { after } \\
\text { first dose }\end{array}$ & $\begin{array}{l}71 \% \\
(33-94 \%)\end{array}$ & $\begin{array}{l}157.30 \\
(41.42-285.23)\end{array}$ & $\begin{array}{l}76 \% \\
(30-100 \%)\end{array}$ & $\begin{array}{l}116.52 \\
(26.92-217.92)\end{array}$ & 5 versus $0^{a}$ & & 0 versus $0^{a}$ & \\
\hline $\begin{array}{l}\text { Days } 7-56 \text { after } \\
\text { second dose }\end{array}$ & $\begin{array}{l}96 \% \\
(89-100 \%)\end{array}$ & $\begin{array}{l}933.40 \\
(685.60-1192.33)\end{array}$ & $\begin{array}{l}97 \% \\
(91-100 \%)\end{array}$ & $\begin{array}{l}621.70 \\
(433.68-847.26)\end{array}$ & $\begin{array}{l}89 \% \\
(43-100 \%)\end{array}$ & $\begin{array}{l}132.28 \\
(31.67-241.03)\end{array}$ & 1 versus $0^{a}$ & \\
\hline
\end{tabular}

RRs and RDs (per 100,000 persons) of COVID-19 outcomes for vaccination versus no vaccination at several time points after vaccination in pregnant women who are members of the CHS, 20 December 2020 through to 3 June 2021. The study population numbered 10,861 individuals in each arm and 1,529 individuals were first included as unvaccinated and then re-recruited as vaccinated. aEstimates were only calculated for cells with more than five events; otherwise, raw counts are reported.

Of the matched individuals, $18 \%$ had at least 1 risk factor for severe COVID-19, the most common being obesity.

During a median follow-up of $77 \mathrm{~d}, 131$ infections were documented in the vaccination group and 235 infections in the control group (Fig. 1). Event counts in each of the analysis periods (for individuals who were eligible to be included in the analysis for that period) are included in Supplementary Table 3. Cumulative incidence curves from baseline (first vaccine dose for the vaccinated group) are shown in Fig. 1 for the documented infection outcome and in Extended Data Figs. 2-4 for the additional outcomes. The curves in the vaccinated and unvaccinated groups are similar until day 14, when incidence in the vaccinated group begins to decline sharply.

The estimated vaccine effectiveness for documented infections was $67 \%(95 \%$ confidence interval $(\mathrm{CI})=40-84 \%)$ in days $14-20$ after the first dose, $71 \%(33-94 \%)$ in days $21-27$ after the first dose and $96 \%(89-100 \%)$ in days 7-56 after the second dose (Table 1). The estimated vaccine effectiveness for symptomatic infection was $66 \%(95 \% \mathrm{CI}=32-86 \%)$ in days $14-20$ after the first dose, $76 \%$ (30-100\%) in days $21-27$ after the first dose and 97\% (91-100\%) in days 7-56 after the second dose. Vaccine effectiveness for COVID-19-related hospitalization was $89 \%$ (43-100\%) in days 7-56 after the second dose. Vaccine effectiveness could not be meaningfully estimated for the other outcomes and time periods due to the small number of events.

\section{Discussion}

In this study, we estimated that the BNT162b2 mRNA COVID-19 vaccine is as effective for pregnant women as previously reported for the general population during the same time period: $96 \%$ effectiveness against documented infection and $97 \%$ effectiveness against symptomatic infection $7-56 \mathrm{~d}$ after receipt of the second vaccine 
dose. The estimated vaccine effectiveness for COVID-19-related hospitalization was high but a paucity of cases prevented precise estimation. These results reflect the effectiveness mainly against the original SARS-CoV-2 reference strain and the B.1.1.7 (Alpha) variant, which were the dominant strains circulating in Israel during the study period.

Since the original phase 3 trials did not include pregnant women, data regarding the effectiveness of the new mRNA COVID-19 vaccines in this population is still scarce in the medical literature. One study confirmed the immunogenicity of these vaccines in pregnant women by confirming cellular and humoral immune responses against SARS-CoV-2 (ref. ${ }^{13}$ ).

We previously estimated the vaccine effectiveness of the BNT162b2 mRNA COVID-19 vaccine for a general population using the data repositories of the same healthcare organization and covering the same variant distribution. After the second vaccine dose, vaccine effectiveness was estimated to be $92 \%$ (88-95\%) for documented infections and 94\% (87-98\%) for symptomatic infections ${ }^{14}$. In an updated analysis of the same data over a longer period, the $95 \%$ CIs were narrower, with estimates of 93\% (91-94\%) for documented infection and $96 \%$ (94-97\%) for symptomatic infection $^{15}$. Our study suggests that the vaccine effectiveness estimate for pregnant women is not lower than that for the general population. This is consistent with the lower prevalence of comorbidities (some of which were associated with lower vaccine effectiveness ${ }^{15}$ ) in pregnant women compared with the general population. Our findings make it plausible that the vaccine effectiveness estimated in the general population for future variants may be used to infer the effectiveness in pregnant women for the same variants, particularly for mRNA-based vaccines.

Vaccination of pregnant women may also provide protection for their newborns. A recent study found binding and neutralizing antibodies in the cord blood of infants born to mothers who were vaccinated with mRNA vaccines and in the mothers' breast milk ${ }^{13}$. Another study found that vaccination of breastfeeding women resulted in a rapid increase of anti-SARS-CoV-2-specific antibodies in their breast milk ${ }^{16}$. The magnitude and duration of this potential protection is still unclear.

The high vaccine effectiveness in pregnant women estimated in this study might also contribute to increased vaccination acceptance rates among this population. It was previously reported that high vaccine effectiveness is an important factor for the encouragement of pregnant women to receive COVID-19 vaccines. If vaccine effectiveness $>90 \%$ was achieved, $52 \%$ of pregnant women reported that they would be willing to receive the vaccine. Furthermore, pregnant women indicated higher likelihood of vaccination with higher vaccine effectiveness ${ }^{12}$.

This study has several limitations. First, despite the careful matching between cohorts, there is the lingering possibility of residual confounding. This is particularly true because information regarding prenatal complications was not available. However, the very similar incidence of documented and symptomatic infections between the two study groups during the early period after the first vaccine dose suggests that residual confounding, if present, was minor. Second, owing to the low incidence of the more severe outcomes, this study could not provide precise vaccine effectiveness estimates for them. Third, the strict matching process required to achieve exchangeability between the study groups resulted in a relatively large fraction of the eligible population not being included in the study. Thus, the proportion of women with some chronic conditions was somewhat lower in the final study population. Vaccine effectiveness for women with chronic conditions may be somewhat lower than the average vaccine effectiveness estimated in this study, as previously reported for the general population ${ }^{15}$.
In conclusion, the results of this study indicate that the BNT162b2 mRNA COVID-19 vaccine is highly effective in pregnant women for the variants circulating in Israel at the time of the study, with vaccine effectiveness that was comparable to that estimated in the general population ${ }^{14}$. Further studies are needed to better characterize the dynamics of vaccine effectiveness throughout pregnancy, the relationship between vaccination timing and infant protection after birth and pregnancy- and non-pregnancy-related safety outcomes.

\section{Online content}

Any methods, additional references, Nature Research reporting summaries, source data, extended data, supplementary information, acknowledgements, peer review information; details of author contributions and competing interests; and statements of data and code availability are available at https://doi.org/10.1038/ s41591-021-01490-8.

Received: 28 June 2021; Accepted: 5 August 2021;

Published online: 7 September 2021

\section{References}

1. Zambrano, L. D. et al. Update: characteristics of symptomatic women of reproductive age with laboratory-confirmed SARS-CoV-2 infection by pregnancy status-United States, January 22-October 3, 2020. MMWR Morb. Mortal. Wkly. Rep. 69, 1641-1647 (2020).

2. Wei, S. Q., Bilodeau-Bertrand, M., Liu, S. \& Auger, N. The impact of COVID-19 on pregnancy outcomes: a systematic review and meta-analysis. CMAJ 193, E540-E548 (2021).

3. BioNTech SE. Study to Evaluate the Safety, Tolerability, and Immunogenicity of SARS CoV-2 RNA Vaccine Candidate (BNT162b2) Against COVID-19 in Healthy Pregnant Women 18 Years of Age and Older https://clinicaltrials.gov/ ct2/show/NCT04754594 (2021).

4. Rubin, R. Pregnant people's paradox-excluded from vaccine trials despite having a higher risk of COVID-19 complications. JAMA 325, 1027-1028 (2021).

5. Rasmussen, S. A., Kelley, C. F., Horton, J. P. \& Jamieson, D. J. Coronavirus Disease 2019 (COVID-19) vaccines and pregnancy: what obstetricians need to know. Obstet. Gynecol. 137, 408-414 (2021).

6. Goodman, T. Update on WHO Interim Recommendations on COVID-19 Vaccination of Pregnant and Lactating Women https://cdn.who.int/media/ docs/default-source/2021-dha-docs/update-on-who-interimrecommendations-on-c-19-vaccination-for-pregnant-and-lactatingwomen-70-.pdf (2021)

7. Centers for Disease Control and Prevention. COVID-19 Vaccines While Pregnant or Breastfeeding https://www.cdc.gov/coronavirus/2019-ncov/ vaccines/recommendations/pregnancy.html (2021).

8. American College of Obstetricians and Gynecologists. COVID-19 Vaccination Considerations for Obstetric-Gynecologic Care https://www.acog.org/clinical/ clinical-guidance/practice-advisory/articles/2020/12/covid-19-vaccinationconsiderations-for-obstetric-gynecologic-care (2021).

9. Shimabukuro, T. T. et al. Preliminary findings of mRNA Covid-19 vaccine safety in pregnant persons. N. Engl. J. Med. 384, 2273-2282 (2021).

10. Riley, L. E. \& Jamieson, D. J. Inclusion of pregnant and lactating persons in COVID-19 vaccination efforts. Ann. Intern. Med. 174, 701-702 (2021).

11. Kourtis, A. P., Read, J. S. \& Jamieson, D. J. Pregnancy and infection. N. Engl. J. Med. 370, 2211-2218 (2014).

12. Skjefte, M. et al. COVID-19 vaccine acceptance among pregnant women and mothers of young children: results of a survey in 16 countries. Eur. J. Epidemiol. 36, 197-211 (2021).

13. Collier, A.-R. Y. et al. Immunogenicity of COVID-19 mRNA vaccines in pregnant and lactating women. JAMA 325, 2370-2380 (2021).

14. Dagan, N. et al. BNT162b2 mRNA Covid-19 vaccine in a nationwide mass vaccination setting. N. Engl. J. Med. 384, 1412-1423 (2021).

15. Barda, N., Dagan, N. \& Balicer, R. D. BNT162b2 mRNA Covid-19 vaccine in a nationwide mass vaccination setting. Reply. N. Engl. J. Med. 384, 1970 (2021).

16. Perl, S. H. et al. SARS-CoV-2-specific antibodies in breast milk after COVID-19 vaccination of breastfeeding women. JAMA 325, 2013-2014 (2021).

Publisher's note Springer Nature remains neutral with regard to jurisdictional claims in published maps and institutional affiliations.

(c) The Author(s), under exclusive licence to Springer Nature America, Inc. 2021 


\section{Methods}

Ethics. This study was approved by the CHS community institutional review board.

Data source. CHS is an integrated healthcare payer-provider organization that serves $52 \%$ of the Israeli population. Medical insurance in Israel is mandatory for all residents, and covers a wide range of services, including prenatal care. The CHS population is fairly representative of the general Israeli population ${ }^{17}$. The present study was based on CHS data covering patients vaccinated from the start of the vaccination campaign in Israel on 20 December 2020 through to 3 June 2021. CHS data systems contain medical and claims data covering all facets of patient care, including primary care, specialist care, imaging, laboratory diagnostics and hospitalizations, with over 20 years of historical depth for most individuals. CHS community care includes dedicated systems for prenatal care, with specific data fields for 'date of last menstrual period' and 'projected birth date'. Pregnancies are recorded in these dedicated systems from the moment a woman begins prenatal care, which is freely and universally available in Israel. These data are integrated daily with data collected centrally by the Israeli Ministry of Health regarding COVID-19 vaccines, SARS-CoV-2 tests and COVID-19-related hospitalizations, disease severity and death.

Study design and study population. We conducted an observational cohort study that emulates a target trial to estimate the effectiveness of the BNT162b2 mRNA COVID-19 vaccine in pregnant women. We used a similar methodology to previous studies on vaccine effectiveness using the same database ${ }^{14}$. Eligibility criteria considered at the start of follow-up were pregnancy (as recorded in the CHS data systems), age of 16 years or older, continuous membership in CHS for 1 complete year, no previous positive SARS-CoV-2 PCR test, no previous SARS-CoV-2 vaccination, not residing in long-term care facilities, no home confinement due to medical reasons, not being a healthcare worker and no interaction with the healthcare system (physician appointment, laboratory test or hospitalization) in the previous $2 \mathrm{~d}$ (since this may signal a preexisting SARS-CoV-2 infection). Individuals with missing data (only relevant for the body mass index and living area variables) were excluded, as these are rare in the CHS data.

Each day during the study period, eligible women vaccinated on that day were individually matched to eligible women who had not yet been vaccinated and who were not previously matched as controls. Matching factors included age (in 3-year bins), trimester of pregnancy, geostatistical living area (corresponding to a small town or a single neighborhood within a large city or city/town of residence when the smaller geostatistical living area was not available), population sector (General Jewish, Arab or Ultraorthodox Jewish), count of influenza vaccinations in the last 5 years (in 2 bins) and existence of at least 1 Centers for Disease Control and Prevention risk factor for severe COVID-19 (ref. ${ }^{18}$ ). Definitions for all variables used in the study are included in Supplementary Table 4

The outcomes studied were: documented SARS-CoV-2 infection, defined as a positive SARS-CoV-2 PCR test; symptomatic SARS-CoV-2 infection (COVID-19), defined as an infection accompanied by the documentation of COVID-19 symptoms in dedicated fields in the outpatient health record (or an infection that warranted hospitalization); COVID-19-related hospitalization; severe COVID-19, as defined by the Israeli Ministry of Health using international criteria ${ }^{19}$; and COVID-19-related death. The outcome date was set to the date of the first positive test for the first two outcomes and the date of first occurrence for the latter outcomes.

Statistical analysis. After matching, we used the Kaplan-Meier estimator to construct cumulative incidence curves. We estimated vaccine effectiveness at different periods after vaccination: days 14-20 after the first dose; days 21-27 after the first dose; and days 7-56 after the second dose. In each period, we restricted the analysis to matched pairs where both members were not censored and had not developed the outcome before the beginning of that period. We then calculated the risk ratio $(\mathrm{RR})$ and risk difference $(\mathrm{RD})$ during that period, with vaccine effectiveness defined as $1-R R$. To estimate the analog of a per-protocol effect, we censored both members of the matched pair when the control received a vaccination but then allowed the control to be re-recruited as a vaccinated individual if a matched control was found. $95 \%$ CIs were estimated using the nonparametric percentile bootstrap method with 1,000 repetitions. Analysis was performed using R v.4.0.4.

Reporting Summary. Further information on research design is available in the Nature Research Reporting Summary linked to this article.

\section{Data availability}

Due to national and organizational data privacy regulations, individual-level data such as those used for this study cannot be shared openly.

\section{Code availability}

The modeling in this paper used R v.4.0.4 and the tidyverse v.1.3.0, survival v.3.2-7, survminer v.0.4.7 and boot v.1.3-27 R packages, all of which are freely available.

\section{References}

17. Cohen, R. \& Rabin, H. Membership in the Health Funds https://www.btl.gov. il/Publications/survey/Documents/seker289/seker 289.pdf (2017).

18. Centers for Disease Control and Prevention. Certain Medical Conditions and Risk for Severe COVID-19 Illness https://www.cdc.gov/coronavirus/2019ncov/need-extra-precautions/people-with-medical-conditions.html (2021).

19. National Institutes of Health. Coronavirus Disease 2019 (COVID-19)

Treatment Guidelines https://www.covid19treatmentguidelines.nih.gov/ (2021).

\section{Acknowledgements}

This study was supported by the Ivan and Francesca Berkowitz Family Living Laboratory Collaboration at Harvard Medical School and Clalit Research Institute. M.L. was supported by the Morris-Singer Foundation.

\section{Author contributions}

S.H.D., B.Y.R. and R.D.B. contributed equally as senior authors to this study. N.D., N.B., T.B.S., C.K., S.H.D., B.Y.R. and R.D.B. conceived and designed the study. N.D., N.B. and M.M.A. participated in data extraction and analysis. N.D., N.B., I.S.K., M.A.H., M.L., S.H.D., B.Y.R. and R.D.B. wrote the manuscript. T.B.S. and S.H.D provided clinical guidance. All authors critically reviewed the manuscript and decided to proceed with publication. B.Y.R. and R.D.B. supervised the study. R.D.B. vouches for the data and analysis.

\section{Competing interests}

N.D., N.B., M.M.A. and R.D.B. report institutional grants to the Clalit Research Institute from Pfizer outside the submitted work and unrelated to COVID-19, with no direct or indirect personal benefits. M.L. reports grants from Pfizer, personal fees from Merck, Bristol Meyers Squibb, Sanofi Pasteur and Janssen, grants from the National Institutes of Health (NIH), the National Institute for Health Research, Centers for Disease Control and Prevention, Open Philanthropy Project, Wellcome Trust and Pfizer outside the submitted work; he has provided unpaid advice on COVID vaccines or vaccine studies to 1Day Sooner (nonprofit), Pfizer, AstraZeneca, Janssen and COVAXX (United Biosciences). M.A.H. reports grants from the NIH and Veterans Affairs, and personal fees from Cytel and ProPublica. S.H.D. reports consulting fees from Roche and UCB outside the submitted work. B.Y.R. reports grants from the NIH outside the submitted work. The other authors declare no competing interests.

\section{Additional information}

Extended data is available for this paper at https://doi.org/10.1038/s41591-021-01490-8.

Supplementary information The online version contains supplementary material available at https://doi.org/10.1038/s41591-021-01490-8.

Correspondence and requests for materials should be addressed to Ran D. Balicer.

Peer review information Nature Medicine thanks Shabir Madhi, Aziz Sheikh and the other, anonymous, reviewer(s) for their contribution to the peer review of this work. Jennifer Sargent was the primary editor on this article and managed its editorial process and peer review in collaboration with the rest of the editorial team.

Reprints and permissions information is available at www.nature.com/reprints. 


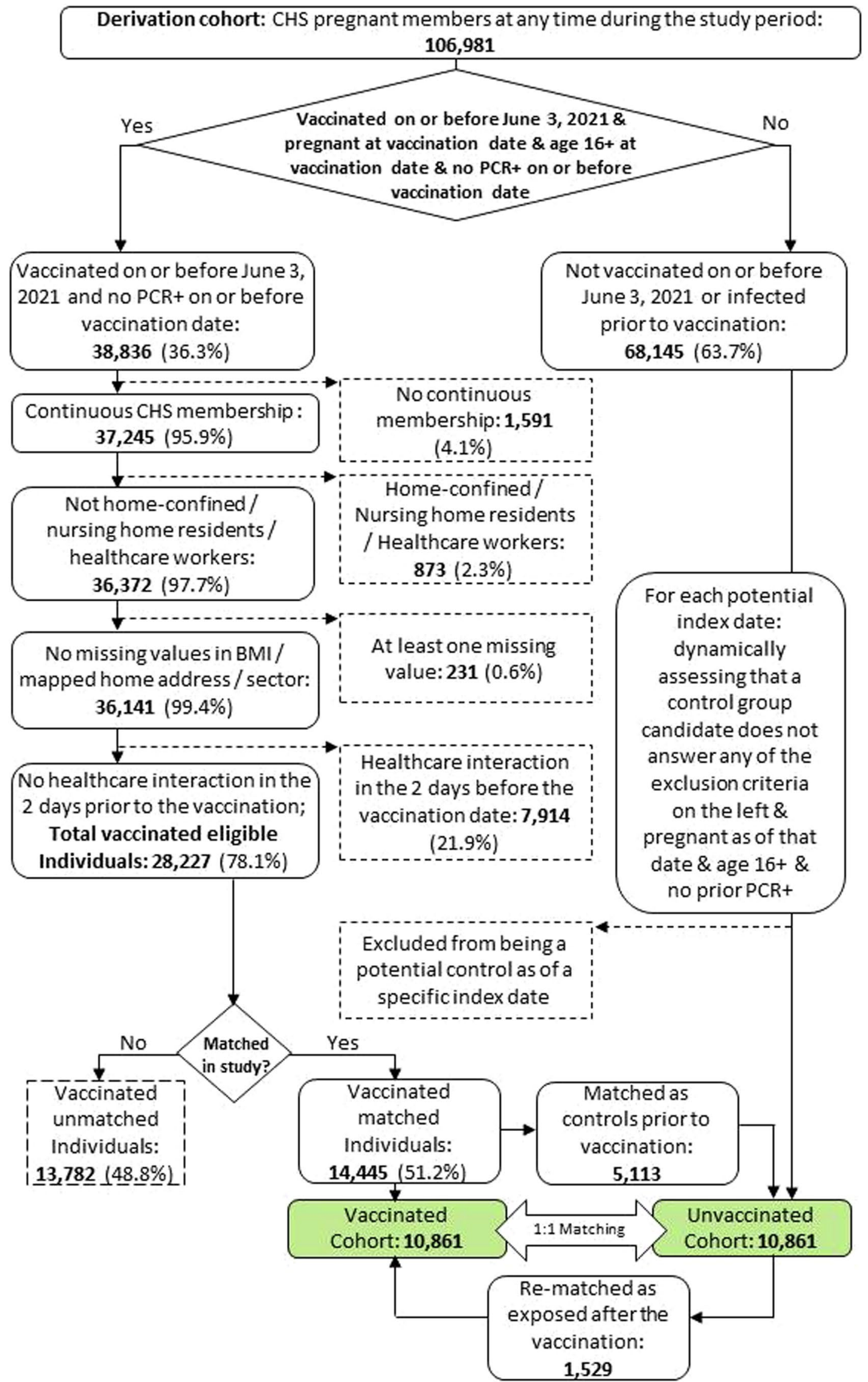

Extended Data Fig. 1 | Population flow chart. Size and percentage change of study population resulting from each inclusion and exclusion criteria. The figure focuses on the vaccinated population. 
Symptomatic SARS-CoV-2 Infection

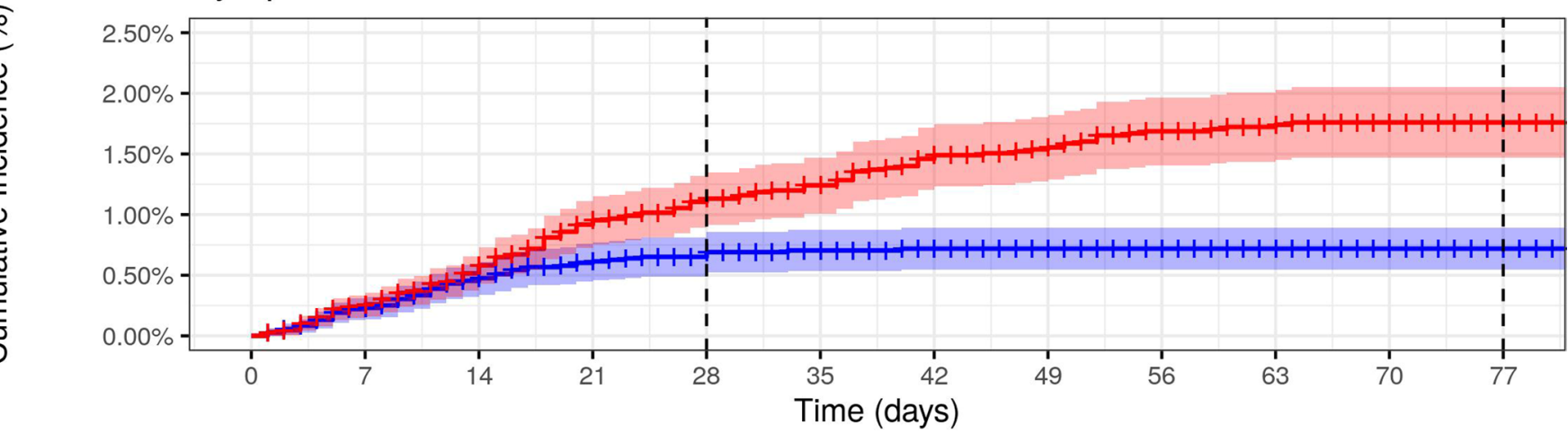

Number at risk
\begin{tabular}{|ccccccccccccc|}
\hline- \\
\hline-10861 & 9905 & 8979 & 8279 & 7631 & 7057 & 6608 & 6159 & 5804 & 5382 & 5000 & 4547 \\
10861 & 9902 & 8970 & 8246 & 7582 & 6999 & 6530 & 6075 & 5709 & 5283 & 4900 & 4446 \\
\hline 1 & 7 & 14 & 21 & 28 & 35 & 42 & 49 & 56 & 63 & 70 & 77 \\
& & & & & Time (days) & & & & &
\end{tabular}

\section{Cumulative number of events}

\begin{tabular}{|c|c|c|c|c|c|c|c|c|c|c|c|c|}
\hline Vaccinated - & 0 & 24 & 47 & 59 & 65 & 66 & 67 & 67 & 67 & 67 & 67 & 67 \\
\hline Unvaccinated - & 0 & 27 & 57 & 89 & 103 & 111 & 128 & 132 & 140 & 143 & 144 & 144 \\
\hline & 0 & 7 & 14 & 21 & 28 & $\begin{array}{c}1 \\
35\end{array}$ & 42 & 49 & 56 & 63 & 70 & 77 \\
\hline
\end{tabular}

Extended Data Fig. 2 | Cumulative incidence of SARS-CoV-2 symptomatic infection in vaccinated pregnant women and matched controls. Cumulative incidence curves of the SARS-CoV-2 symptomatic infection outcome in pregnant women, Clalit Health Services, December 20, 2020 through June 3, 2021. The main line is the point estimate of the cumulative incidence and the shaded areas are $95 \%$ confidence intervals. The vertical lines at days 28 and 77 demarcate the period in which an individual is considered 'fully vaccinated' in the manuscript ( 7 through 56 days after receipt of the second dose). The table below the curve presents the number at risk at each time point, along with the cumulative number of events. Each individual was included only once in each study group, but individuals could move from the unvaccinated group to the vaccinated group after receipt of the vaccine. 
COVID-19 Hospitalization

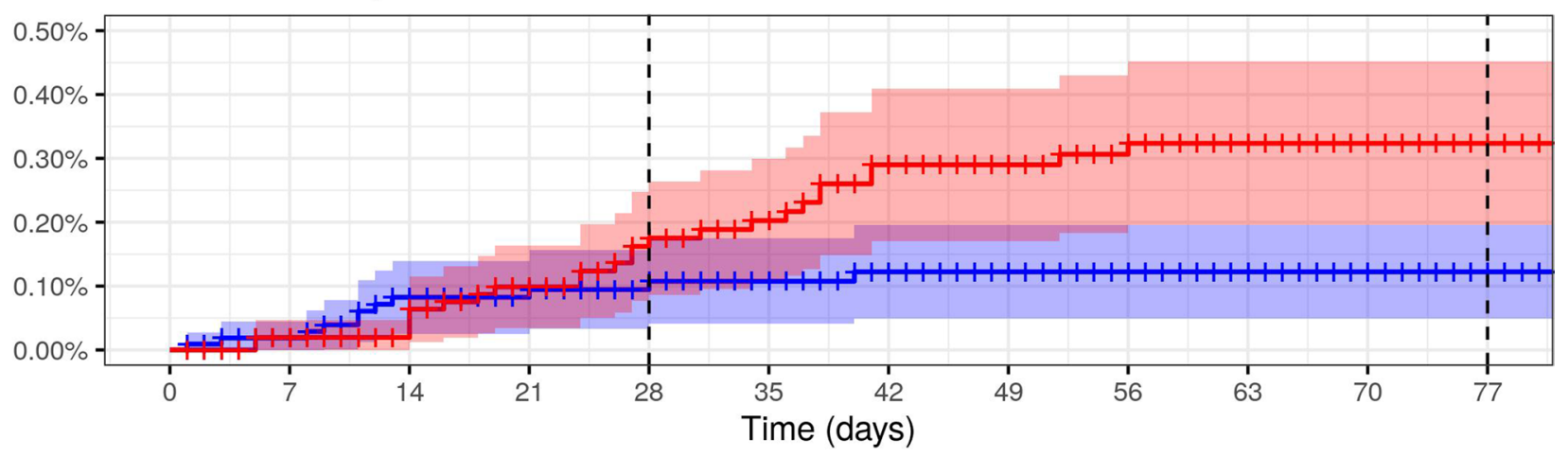

\begin{tabular}{|c|c|c|c|c|c|c|c|c|c|c|c|}
\hline 10861 & 9925 & 9013 & 8324 & 7675 & 7101 & 6648 & 6198 & 5841 & 5417 & 5035 & 4580 \\
\hline 10861 & 9925 & 9019 & 8323 & 7669 & 7093 & 6633 & 6183 & 5825 & 5400 & 5018 & 4563 \\
\hline 0 & 7 & 14 & 21 & 28 & $\begin{array}{c}35 \\
\text { Time }\end{array}$ & $\begin{array}{c}42 \\
\text { days) }\end{array}$ & 49 & 56 & 63 & 70 & 77 \\
\hline
\end{tabular}

\section{Cumulative number of events}

\begin{tabular}{|c|c|c|c|c|c|c|c|c|c|c|c|c|}
\hline Vaccinated - & 0 & 2 & 8 & 9 & 10 & 10 & 11 & 11 & 11 & 11 & 11 & 11 \\
\hline Unvaccinated - & 0 & 2 & 6 & 9 & 15 & 17 & 23 & 23 & 25 & 25 & 25 & 25 \\
\hline & 0 & 7 & 14 & 21 & 28 & 35 & 42 & 49 & 56 & 63 & 70 & 77 \\
\hline
\end{tabular}

Extended Data Fig. 3 | Cumulative incidence of COVID-19 hospitalization in vaccinated pregnant women and matched controls. Cumulative incidence curves of the COVID-19 hospitalization outcome in pregnant women, Clalit Health Services, December 20, 2020 through June 3, 2021. The main line is the point estimate of the cumulative incidence and the shaded areas are $95 \%$ confidence intervals. The vertical lines at days 28 and 77 demarcate the period in which an individual is considered 'fully vaccinated' in the manuscript ( 7 through 56 days after receipt of the second dose). The table below the curve presents the number at risk at each time point, along with the cumulative number of events. Each individual was included only once in each study group, but individuals could move from the unvaccinated group to the vaccinated group after receipt of the vaccine. 


\section{Severe COVID-19}

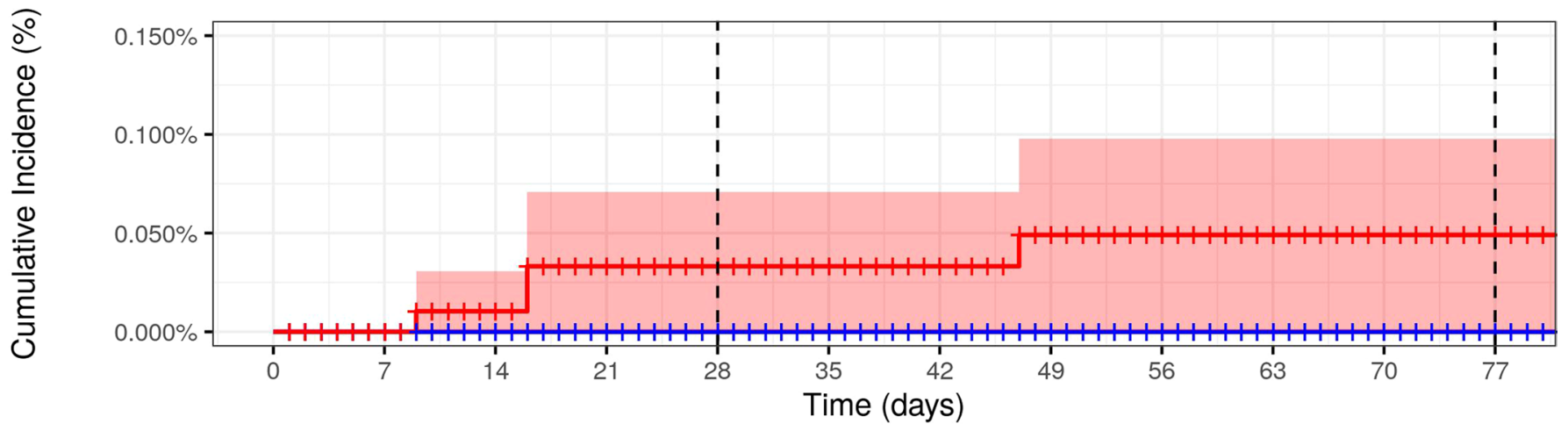

Number at risk
-\begin{tabular}{|ccccccccccccc|}
\hline 10861 & 9927 & 9021 & 8332 & 7683 & 7110 & 6656 & 6206 & 5849 & 5424 & 5042 & 4587 \\
10861 & 9927 & 9020 & 8329 & 7680 & 7107 & 6653 & 6202 & 5845 & 5420 & 5038 & 4583 \\
\hline & 1 & 14 & 21 & 28 & 35 & 42 & 49 & 56 & 63 & 70 & 77 \\
\hline
\end{tabular}

\section{Cumulative number of events}

\begin{tabular}{|c|c|c|c|c|c|c|c|c|c|c|c|c|}
\hline Vaccinated - & 0 & 0 & 0 & 0 & 0 & 0 & 0 & 0 & 0 & 0 & 0 & 0 \\
\hline \multirow{2}{*}{ Unvaccinated - } & 0 & 0 & 1 & 3 & 3 & 3 & 3 & 4 & 4 & 4 & 4 & 4 \\
\hline & 0 & 7 & 14 & 21 & 28 & Tim & $\begin{array}{l}42 \\
\text { lys) }\end{array}$ & 49 & 56 & 63 & 70 & 77 \\
\hline
\end{tabular}

Extended Data Fig. 4 | Cumulative incidence of severe COVID-19 in vaccinated pregnant women and matched controls. Cumulative incidence curves of the severe COVID-19 outcome in pregnant women, Clalit Health Services, December 20, 2020 through June 3, 2021. The main line is the point estimate of the cumulative incidence and the shaded areas are $95 \%$ confidence intervals. The vertical lines at days 28 and 77 demarcate the period in which an individual is considered 'fully vaccinated' in the manuscript ( 7 through 56 days after receipt of the second dose). The table below the curve presents the number at risk at each time point, along with the cumulative number of events. Each individual was included only once in each study group, but individuals could move from the unvaccinated group to the vaccinated group after receipt of the vaccine. 


\section{Reporting Summary}

Nature Portfolio wishes to improve the reproducibility of the work that we publish. This form provides structure for consistency and transparency in reporting. For further information on Nature Portfolio policies, see our Editorial Policies and the Editorial Policy Checklist.

\section{Statistics}

For all statistical analyses, confirm that the following items are present in the figure legend, table legend, main text, or Methods section.

$\mathrm{n} / \mathrm{a} \mid$ Confirmed

$\bigotimes$ The exact sample size $(n)$ for each experimental group/condition, given as a discrete number and unit of measurement

$\square$ \ A statement on whether measurements were taken from distinct samples or whether the same sample was measured repeatedly

Х The statistical test(s) used AND whether they are one- or two-sided

Xnly common tests should be described solely by name; describe more complex techniques in the Methods section.

$\bigotimes$ A description of all covariates tested

Х $\square$ A description of any assumptions or corrections, such as tests of normality and adjustment for multiple comparisons

$\triangle$ A full description of the statistical parameters including central tendency (e.g. means) or other basic estimates (e.g. regression coefficient) AND variation (e.g. standard deviation) or associated estimates of uncertainty (e.g. confidence intervals)

W For null hypothesis testing, the test statistic (e.g. $F, t, r$ ) with confidence intervals, effect sizes, degrees of freedom and $P$ value noted Give $P$ values as exact values whenever suitable.

Х $\square$ For Bayesian analysis, information on the choice of priors and Markov chain Monte Carlo settings

$\bigotimes \square$ For hierarchical and complex designs, identification of the appropriate level for tests and full reporting of outcomes

$\bigotimes \square$ Estimates of effect sizes (e.g. Cohen's $d$, Pearson's $r$ ), indicating how they were calculated

Our web collection on statistics for biologists contains articles on many of the points above.

\section{Software and code}

Policy information about availability of computer code

Data collection SQL Server

Data analysis R version 4.0.4, with packages tidyverse (1.3.0), survival (3.2-7), survminer (0.4.7) and boot (1.3-27)

For manuscripts utilizing custom algorithms or software that are central to the research but not yet described in published literature, software must be made available to editors and

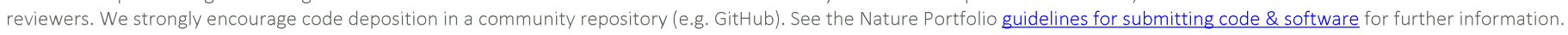

\section{Data}

Policy information about availability of data

All manuscripts must include a data availability statement. This statement should provide the following information, where applicable:

- Accession codes, unique identifiers, or web links for publicly available datasets

- A description of any restrictions on data availability

- For clinical datasets or third party data, please ensure that the statement adheres to our policy 
Please select the one below that is the best fit for your research. If you are not sure, read the appropriate sections before making your selection.

$\square$ Life sciences $\quad \bigotimes$ Behavioural \& social sciences $\quad \square$ Ecological, evolutionary \& environmental sciences

For a reference copy of the document with all sections, see nature.com/documents/nr-reporting-summary-flat.pdf

\section{Behavioural \& social sciences study design}

All studies must disclose on these points even when the disclosure is negative.

Study description

This is a retrospective cohort analysis, based on existing medical records. Methods are strictly quantitative.

Research sample

The population of this study is the patient population of a large integrated healthcare provider operating in Israel. Women who were pregnant during the study period (December 20, 2020 through June 3, 2021) were included. The age median (IQR) was 30 (26-33). This is the full population of the health organization, and is considered representative of the Israeli population as a whole.

Sampling strategy

Due to the retrospective nature of this study all qualified members were included and no sample-size calculation was done.

Data collection

All data used in this study is based on existing electronic medical records. No dedicated data collection was performed. Access to the data was performed through Microsoft SQL Server. The researcher was not blinded to the experimental hypothesis.

Timing

Individuals who were pregnant from December 20, 2020 until June 3, 2021 were eligible. Background variables were extracted from the period prior to inclusion in the study.

Data exclusions

As detailed in Figure S1, individuals were excluded if they were not continuous members, if they were home-confined due to medical reasons, if they were nursing home residents, if they were healthcare workers, if they had missing data for BMI, population sector or home address, if they had a healthcare interaction in the 2 days prior to start of follow-up.

Of eligible individuals, only those that successfully matched were included in the final study cohort.

Eligibility criteria were pre-established.

Non-participation $\quad$ As detailed in Figure S1, the original cohort (pregnant, age 16+, no prior SARS-CoV-2 diagnosis) numbered 38,836. Of these, 28,227 were eligible. Of these, 14,445 matched into the study cohort.

Randomization

This is an observational study, no randomization was performed.

\section{Reporting for specific materials, systems and methods}

We require information from authors about some types of materials, experimental systems and methods used in many studies. Here, indicate whether each material, system or method listed is relevant to your study. If you are not sure if a list item applies to your research, read the appropriate section before selecting a response.

Materials \& experimental systems

$\mathrm{n} / \mathrm{a}$ Involved in the study

Х $\square$ Antibodies

Х $\square$ Eukaryotic cell lines

\ $\square$ Palaeontology and archaeology

\ $\square$ Animals and other organisms

$\square$ \uman research participants

Х Clinical data

$\bigotimes \square$ Dual use research of concern

\begin{tabular}{|c|c|}
\hline $\mathrm{n} / \mathrm{a}$ & Involved in the study \\
\hline$\triangle$ & $\square$ ChIP-seq \\
\hline Х & $\square$ Flow cytometry \\
\hline Х & $\square$ MRI-based neuroimaging \\
\hline
\end{tabular}

\section{Human research participants}

Policy information about studies involving human research participants

\section{Population characteristics See above}

Recruitment

Participants were not recruited but rather selected from a retrospective Clalit medical database. All eligible participants were considered.

Ethics oversight

This study was approved by the Clalit research ethics committee. 